\title{
IUE INVESTIGATIONS AT THE CORE OF M 79
}

\author{
Bruce Altner \\ Applied Research Corporation
}

As a result of their investigation of 27 galactic globular clusters with the ANS satellite van Albada, de Boer and Dickens (1981) classified M 79 (NGC 1904) as an "extremely blue" cluster. It was also found to be a low luminosity $x$-ray source based on data acquired with the Einstein X-ray Observatory (Grindlay 1981). In this brief paper we discuss the stellar spectra extracted from two short wavelength (SWP) IUE images acquired at the "center of light" of $M 79$. Discrete peaks in the "spatially resolved", cross-dispersion profile suggested the presence of at least three hot stars in the large aperture in both images (Fig. 1). The images in question are SWP 25303 and SWP 28936 for which it is important to note here that 1 ) the orientation of the large aperture on the plane of the sky differs for the two images by nearly $180^{\circ}$ and 2) the target coordinates, determined from offset maneuvers with respect to a nearby SAO star, are nearly coincident. The cross-dispersion profile observed in the first spectrum is thus repeated in the second case, but is "flipped" left for right. Features immediately apparent in both images include a broad, asymmetric peak, almost certainly the blend of two or more components, and a second peak well separated from this blend and most likely a single star. In Fig. 2 we show the cross-dispersion profile of SWP 28936 and the best fit to that profile, assuming three components. From fits such as this and similar ones in other wavelength bins, we ultimately obtain from each image the entire SWP spectrum of each individual component.

The spectrum of the single source in each image was readily separated from the broader, blended profile. These two spectra are remarkably similar and it is most likely that they are spectra of the same star; we shall refer to this source as star "A". The mean spectrum of Star A is shown in Fig. 3, along with the Kurucz (1979) model atmosphere with $\mathrm{T}_{\text {eff }}=13,000 \mathrm{~K}$ and $\log \mathrm{g}=4.5$, and solar abundances. The IUE standard star HD 29335 ( 49 Eri; B7 V) is a close match to this spectrum. Not so easily resolved were the stars comprising the broad blend, due to the small separation of the peak centers ( $\sim 5^{\prime \prime .5}$ in both images), but we finally were able to achieve reasonable results for the brighter component, which we refer to as star "B"; it is best fit with a Kurucz solar abundance model of $T_{\text {eff }}$ $11,000 \mathrm{~K}$ and $\log \mathrm{g}=2.0$. In this case we find that a B9 IV standard 501

J. E. Grindlay and A. G. Davis Philip (eds.),

The Harlow-Shapley Symposium on Globular Cluster Systems in Galaxies, 501-502.

(C) 1988 by the IAU. 
star $(\alpha$ Del) is a fairly close match to star B's IUE spectrum. Given the position angle and location of the aperture we tentatively identify star A as No. 214 and $B$ as No. 210 from the list of Cordoni and Aurière (1983; $\mathrm{V}=16.8,16.0$ and $(\mathrm{B}-\mathrm{V})=-0.2,0.2$, respectively). From the fits to the Kurucz models we derive $L / L_{0}=2.1$ and 2.5 for stars $A$ and $B$ respectively, indicating an above horizontal-branch location in the cluster color-magnitude diagram.

Investigation of Fig. 1 reveals a startling inconsistency in the above arguments, however it is clear that the separation between Star A and star $B$ is not the same for both images. If real, such a shift would imply a tangential velocity nearly half of the speed of light, which is clearly unreasonable and suggests that perhaps these aren't the same stars at all. Yet, if one considers the total flux in all three components for each image (so that no errors due to the extraction procedure are involved) one finds remarkable agreement between the two (composite) spectra. Perhaps this close agreement is merely fortuitous; with only two images it is difficult to tell, but work continues on this and other questions in $M 79$ and other galactic globular clusters.
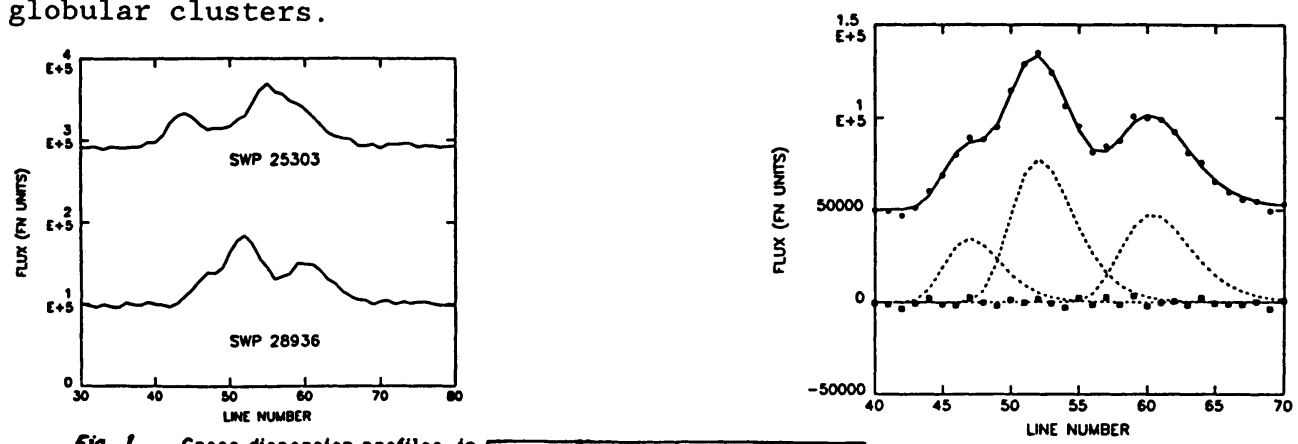
Fig. 1 Cross dispersion profiles in wavelength interval $\lambda$ 1275-1300 A.

Fig. 3. Mean spectrum of Star ${ }^{-A}$ and the Kurucz model (heavy line) with $T_{\text {eff }}=13,000 K, \log g=4.5, \log A=0.0$.

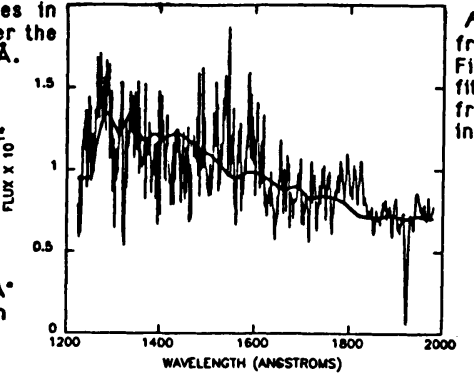
Fig 2. Filled circles are IUE data from SWP 28936, in the same bin as Figure 1; solid line is best composite fit; filled squares show the deviations from the fit; dotted lines are the individual component best fit profiles.

\section{REFERENCES}

Cordoni, J. -P. and Aurière, M. 1983 Astron Astrophys. Supp. $54,431$.

Grindlay, J. E. 1981 in X-Ray Astronomy with the Einstein

Satellite, R. Giacconi, ed., Reidel, Dordrecht, p. 79.

Kurucz, R. L. 1979 Astrophys. J. Suppl. 40, 1.

van Albada, T. S., de Boer, K. S. and Dickens, R. J. 1981

Monthly Notices Roy. Astron. Soc. 195, 591. 